\title{
A NOTE ON THE RELATIONSHIPS BETWEEN CONVEXITY AND INVEXITY
}

\author{
GIORGIO GIORGI
}

(Received 16 November 1988; revised 8 August 1989)

\begin{abstract}
Using the fact that a differentiable quasi-convex function is also pseudo-convex at every point $x$ of its domain where $\nabla f(x) \neq 0$, recent results relating different forms of convexity and invexity are strengthened.
\end{abstract}

In [1] Ben-Israel and Mond provide the following simple and nice characterisation of invex functions:

THeOREM 1. Let $f: R^{n} \rightarrow R$ be differentiable. Then $f$ is invex if and only if every stationary point is a global minimum.

For other proofs of this statement, see [2, 4]. On page 4 of the same article the authors present a diagram showing the various relationships between convex, pseudo-convex, invex, quasi-convex and quasi-invex functions. This diagram can be improved by making use of the following result, due to Crouzeix and Ferland [3].

THEOREM 2. Let $f$ be a differentiable and quasi-convex function on an open convex set $X \subseteq R^{n}$. Then $f$ is pseudo-convex on $X$ if and only if $f$ has a minimum at $x \in X$ whenever $\nabla f(x)=0$.

We provide a new and perhaps simpler proof of this result. The necessary part of the theorem follows from the definition of pseudo-convex functions

${ }^{1}$ Department of Management Researches, Section of General and Applied Mathematics, University of Pavia, 27100 Pavia (Italy).

The author thanks Prof. B. Mond who suggested this note.

(C) Copyright Australian Mathematical Society 1990, Serial-fee code 0334-2700/90 
(see [5]). As for sufficiency, let $x^{0} \in X, \nabla f\left(x^{0}\right)=0 \Rightarrow x^{0}$ is a (global) minimum point of $f(x)$ on $X$, i.e. $\left(x-x^{0}\right)^{\prime} \nabla f\left(x^{0}\right)=0 \Rightarrow f(x) \geqq$ $f\left(x^{0}\right), \forall x \in X$.

It is obvious that $f(x)$ is then locally pseudo-convex at $x^{0}$, with respect to $X$ (see [5]). Let us now prove that: $f(x)$ quasi-convex on $X ; x^{0} \in$ $X ; \nabla f\left(x^{0}\right) \neq 0 \lambda$ implies $f(x)$ pseudo-convex at $x^{0}$, i.e. $\left(x-x^{0}\right)^{\prime} \nabla f\left(x^{0}\right) \geq$ $0 \Rightarrow f(x) \geq f\left(x^{0}\right), \forall x \in \bar{X}$.

Let us consider a point $x^{1} \in X$ such that

$$
\left(x^{1}-x^{0}\right)^{\prime} \nabla f\left(x^{0}\right) \geq 0
$$

but for which it is

$$
f\left(x^{1}\right)<f\left(x^{0}\right) .
$$

Thus $x^{1}$ belongs to the non void set

$$
X_{0}=\left\{x \mid x \in X, f(x) \leq f\left(x^{0}\right)\right\},
$$

whose elements, thanks to the quasi-convexity of $f(x)$, verify the relation

$$
x \in X_{0} \Rightarrow\left(x-x^{0}\right)^{\prime} \nabla f\left(x^{0}\right) \leq 0 .
$$

Let us now consider the sets, both non void,

$$
W=\left\{x \mid x \in X,\left(x-x^{0}\right)^{\prime} \nabla f\left(x^{0}\right) \geq 0\right\}, \quad X_{00}=X_{0} \cap W .
$$

The following implication obviously holds:

$$
x \in X_{00} \Rightarrow x \in H_{0}=\left\{x \mid x \in X,\left(x-x^{0}\right)^{\prime} \nabla f\left(x^{0}\right)=0\right\} .
$$

It is therefore evident that $X_{00}$ is included in the hyperplane (since $\nabla f\left(x^{0}\right) \neq$ 0) $H=\left\{x \mid x \in R^{n},\left(x-x^{0}\right)^{\prime} \nabla f\left(x^{0}\right)=0\right\}$, a hyperplane supporting $X_{0}$, owing to (3). Relations (1) and (2) point out that $x^{1}$ belongs to $W$ and $X_{0}$ and hence to $X_{00}, H_{0}, H$. Moreover (2) says that $x^{1}$ lies in the interior of $X_{0}$ : therefore $x^{1}$ at the same time belongs to the interior of a set and to a hyperplane supporting the same set, which is absurd. So relation (2) is false and (1) implies $f\left(x^{1}\right) \geq f\left(x^{0}\right)$.

The quasi-convex function $f(x)$ is thus pseudo-convex at every point $x$ of $X$ where $\nabla f(x) \neq 0$. Consequently we note that sufficient conditions to test the quasi-convexity of a function, in a convex set where $\nabla f(x) \neq$ $0, \forall x \in X$, really locate the class of pseudo-convex functions. This is, for example, the case of the determinantal conditions for twice continuously differentiable functions established by Arrow and Enthoven and generalised by other authors. (see [3]). 


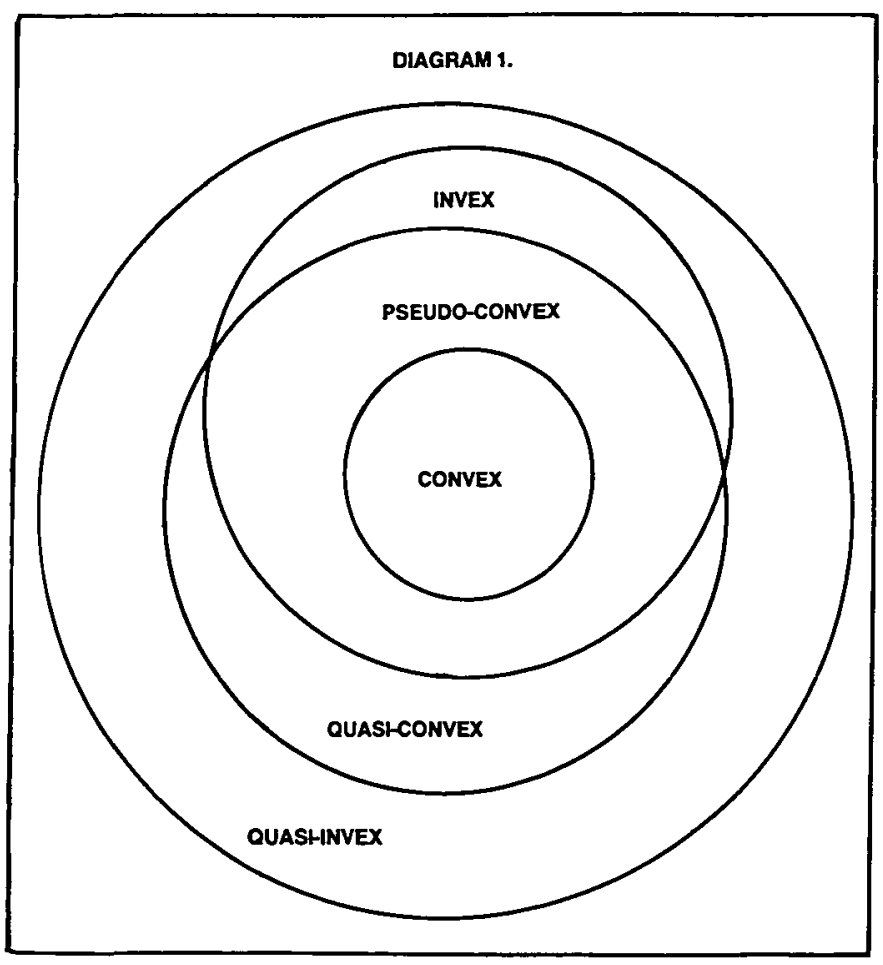

Taking Theorem 2 into account, the diagram on page 4 in [1] must be modified as above.

\section{References}

[1] A. Ben-Israel and B. Mond, "What is invexity?", J. Austral. Math. Soc. Ser. B 28 (1986) 1-9.

[2] B. D. Craven and B. M. Glover, "Invex functions and duality", J. Austral. Math. Soc. Ser. A 39 (1985) 1-20.

[3] J. P. Crouzeix and J. A. Ferland, "Criteria for quasiconvexity and pseudoconvexity: relationships and comparisons", Math. Programming 23 (1982) 193-205.

[4] V. Jeyakumar, "Strong and weak invexity in mathematical programming", Methods Oper. Res. 55 (1985) 109-125.

[5] O. L. Mangasarian, Nonlinear Programming (McGraw-Hill, New York, 1969). 